\title{
Phase-coherent effects in multiterminal superconductor/normal metal mesoscopic structures
}

\author{
A.F. Volkov*† and R. Seviour*, \\ * School of Physics and Chemistry, \\ Lancaster University, Lancaster LA1 4 YB, U.K. \\ ${ }^{\dagger}$ Institute of Radioengineering and Electronics of the Russian \\ Academy of Sciencies, Mokhovaya str.11, Moscow 10390\%, Russia.
}

(November 20, 2018)

\begin{abstract}
In this report we analyse three effects which may arise in a mesoscopic multiterminal $\mathrm{S} / \mathrm{N}$ structure (two normal and two superconducting reservoirs). We show that the Josephson critical current is a non-monotonic function of a voltage between the normal reservoirs. The influence of the spin-polarized electrons on the Josephson critical current was also studied. We show that if there is a temperature difference between the normal reservoirs, a voltage between the superconductors and normal conductors arises which oscillates as the phase difference varies. Its magnitude is much greater than the thermoemf in the case of the ordinary thermoelectric effect.
\end{abstract}

\section{Introduction}

In this report we present results of the theoretical study of transport in diffusive 4-terminal superconductor-normal metal $(\mathrm{S} / \mathrm{N})$ mesoscopic structures. A great deal of attention was paid recently to the problem of the influence a current between the $\mathrm{N}$ reservoirs has on the Josephson effect in the superconducting curcuit. This problem was analysed first in Ref. [1] for a ballistic S/N system and in Ref. [2] for a diffusive system. It was shown that the Josephson critical current $I_{c}$ changes sign at a certain voltage $V$ between the $\mathrm{N}$ reservoirs, and the Josephson junction switches into a $\pi$-state ( $\pi$-junction). The reason for the sign reversal effect is that the distribution function in the $\mathrm{N}$ wire, which can have an equilibrium form, is shifted with respect to the distribution function in the superconductors by the value $V$. Later the dependence $I_{c}(V)$ was calculated in various models and approximations in Refs. [3]5-8] (diffusive case) and [9.10] (ballistic case). The sign reversal effect in a diffusive $\mathrm{Au} / \mathrm{Nb}$ mesoscopic structure was observed by Baselmans et al. [11].

In multi-terminal $\mathrm{S} / \mathrm{N}$ structures one can observe not only the sign reversal effect, but also a number of other interesting phenomena. It was shown in Refs. [3] that the measured critical current $I_{m}$ in a structure similar to that shown in Fig.1a may not only decrease, but also may increase with increasing voltage $V$. In particular one can observe the Josephson-like effects (plateau on the $I_{3}\left(V_{S}\right)$ curve, oscillations of the measured critical current $I_{m}$ in a magnetic field etc) even if the Josephson coupling between superconductors under equilibrium conditions is vanishing. The stimulation of the Josephson effect in this structure is related in particular to a phase dependence of the $\mathrm{S} / \mathrm{N}$ interface or $\mathrm{N}$ wire conductance: $\delta G \sim \cos \varphi$. It turns out that apart from the Josephson term $I_{c} \sin \varphi$, an additional term $I_{s g} \cos \varphi$ appears in a dynamic equation for $\varphi$. This term leads to a change of the critical current from the value $I_{c}$ to a new (measured) value $I_{m}=\sqrt{I_{c}^{2}+I_{s g}^{2}}$, where $I_{s g}$ is the amplitude of the subgap current. The stimulation of the Josephson effect by a current between the $\mathrm{N}$ reservoirs was analysed in Refs. [3] on a simplified model of gapless superconductors. Here we present results of the analysis for the real case where the superconductors have a gap and the density-of-states (DOS) has a singularity [4. We show that the measured critical current $I_{m}$ reaches a maximum at $e V \cong \Delta$ and depends weakly on the temperature $T$. These results agree with recent observations of the stimulation of the Josephson critical current 12].

We also study the effect of the spin injection into the $\mathrm{N}$ wire from the ferromagnetic $(\mathrm{F})$ reservoirs on the critical current $I_{c}$ for the structure shown in Fig.1b. If the magnetizations in the F reservoirs have the same direction, then there is no spin injection. In the case of antiparallel orientation, the total magnetization of the injected electrons in the $\mathrm{N}$ wire is finite. We show that in the case of a $\mathrm{N}$ wire short length $2 L\left(L<\left\{L_{\epsilon}, L_{s p}\right\}\right.$; here $L_{\epsilon}$ and $L_{s p}$ are the energy and spin relaxation length) the spin injection does not affect the critical current and the dependence $I_{c}(V)$ is the same as in the case of normal (nonmagnetic) reservoirs. In a semi-mesoscopic structure $\left(L_{\epsilon}<L<L_{s p}\right)$ the $\pi$-junction is realised only in the case of the antiparallel orientation of the magnetizations in the $\mathrm{F}$ reservoirs when spins are accumulated in the $\mathrm{N}$ wire.

Finally we analyse the thermoelectric effect in the structure shown in Fig.1a. We assume that there is no current between the $\mathrm{N}$ reservoirs, but the reservoirs are maintained at different temperatures $T=T_{o} \pm \delta T$. It will be shown that the temperature gradient leads to a voltage difference $V_{T}$ between the normal and superconducting circuits. This voltage does not contain the small parameter $T / \epsilon_{F}$ (as is the case for the ordinary thermoeffect in normal metals) and reaches a value of order $\delta T / e$. In addition, the voltage $V_{T}$ depends on the phase difference $\varphi$; it is zero at $\varphi=0$ 
and oscillates with increasing $\varphi$.

\section{Basic equations}

The Green's function technique is perhaps the most convenient and powerful method for studing transport and nonequlibrium phenomena in $\mathrm{S} / \mathrm{N}$ mesoscopic structures. If we are not interested in purely quantum interference effects arising as the result of multiple (non-Andreev) reflections, then we can use a simpler, well developed method of quasiclassical Green's functions (see, for example, [13]). In this method the matrix Keldysh function $\widehat{G}$ is introduced along with the retarded (advanced) matrix Green's functions $\widehat{G}^{R(A)}=G^{R(A)} \widehat{\tau}_{z}+\widehat{F}^{R(A)}$, here $\widehat{F}^{R(A)}$ is the matrix condensate Green's function. The functions $\widehat{G}^{R(A)}$ determine the excitation spectrum and the DOS. The function $\widehat{G}$ describes the nonequilibrium properties and is related to the matrix distribution functions $\widehat{f}: \widehat{G}_{\alpha}=\widehat{G}_{\alpha}^{R} \widehat{f}_{\alpha}-\widehat{f} \widehat{G}_{\alpha} \widehat{G}_{\alpha}^{A}$, where $\widehat{f}_{\alpha}=f_{\alpha+} \widehat{1}+f_{\alpha-} \widehat{\tau}_{z}$. The components of $\widehat{f}_{\alpha}$ can be expressed through the distribution functions of electrons $n_{\alpha}$ and holes $p_{\alpha}$ ( $\alpha$ is the spin index). The function $f_{\alpha+}$ describes an electron and hole distribution symmetrical in branch populations: $f_{\alpha+}=1-\left(n_{\alpha}+p_{\bar{\alpha}}\right)$, here $\bar{\alpha}=\downarrow$ if $\alpha=\uparrow$. The function $f_{\alpha-}=-\left(n_{\alpha}-p_{\bar{\alpha}}\right)$ describes the branch imbalance and determines the electric potential.

The equations for $\widehat{G}_{\alpha}$ and $\widehat{G}_{\alpha}^{R(A)}$ can be solved analitically in the case of a short structure $\left(\epsilon_{T h} \equiv D / L^{2}>>\epsilon\right.$, where $\epsilon$ is a characteristic energy; $\epsilon \cong \min \{T, \Delta\}$ ) [14 18]. In the general case one needs to solve equations for the distribution functions $f_{\alpha \pm}$ and the Usadel equation [17,19 21]. In the present paper we consider the case when the temperature may be both greater or less than the Thouless energy $\epsilon_{T h}$ and the approximation of a short length is not valid. The distrubution functions in the structure shown in Fig.1a obey the kinetic equation (we consider only the dirty limit)

$$
L \partial_{x}\left[M_{ \pm} \partial_{x} f_{ \pm}(x)+J_{S} f_{\mp}(x) \pm J_{a n} \partial_{x} f_{\mp}(x)\right]=r\left[A_{ \pm} \delta\left(x-L_{1}\right)+\overline{A_{ \pm}} \delta\left(x+L_{1}\right)\right] .
$$

where $\quad M_{ \pm}=\left(1-G^{R} G^{A} \mp\left(\widehat{F}^{R} \widehat{F}^{A}\right)_{1}\right) / 2 ; J_{a n}=\left(\widehat{F}^{R} \widehat{F}^{A}\right)_{z} / 2, J_{s}=(1 / 2)\left(\widehat{F}^{R} \partial_{x} \widehat{F}^{R}-\widehat{F}^{A} \partial_{x} \widehat{F}^{A}\right)_{z}, A_{ \pm}=\left(\nu \nu_{S}+\right.$ $\left.g_{1 \mp}\right)\left(f_{ \pm}-f_{S \pm}\right)-\left(g_{z \pm} f_{S \mp}+g_{z \mp} f_{\mp}\right) ; g_{1 \pm}=(1 / 4)\left[\left(\widehat{F}^{R} \pm \widehat{F}^{A}\right)\left(\widehat{F}_{S}^{R} \pm \widehat{F}_{S}^{A}\right)\right]_{1} ; g_{z \pm}=(1 / 4)\left[\left(\widehat{F}^{R} \mp \widehat{F}^{A}\right)\left(\widehat{F}_{S}^{R} \pm \widehat{F}_{S}^{A}\right)\right]_{z}$;

The parameter $r=R / R_{b}$ is the ratio of the resistance of the $\mathrm{N}$ wire $R$ and $\mathrm{S} / \mathrm{N}$ interface resistance $R_{b}$; the functions $\overline{A_{-}}$and $\bar{A}_{+}$coincide with $\overline{A_{-}}, \bar{A}_{+}$if we make a substitution $\varphi \rightarrow-\varphi$. We introduced above the following notations $\left(\widehat{F}^{R} \widehat{F}^{A}\right)_{1}=\operatorname{Tr}\left(\widehat{F}^{R} \widehat{F}^{A}\right),\left(\widehat{F}^{R} \widehat{F}^{A}\right)_{z}=\operatorname{Tr}\left(\widehat{\tau}_{z} \widehat{F}^{R} \widehat{F}^{A}\right)$ etc.; $\nu, \nu_{S}$ are the DOS in the $\mathrm{N}$ film at $x=L_{1}$ and in the superconductors. The functions $f_{S \pm}$ are the distribution functions in the superconductors which are assumed to have equilibrium forms. This means that $f_{S+} \equiv f_{e q}=\tanh (\epsilon \beta)$ (here $\beta=1 /(2 T)$ ) and $f_{S_{-}}=0$, because we set the potential of the superconductors equal to zero (no branch imbalance in the superconductors). We assumed that the width of the $\mathrm{S} / \mathrm{N}$ interface $w$ is much less than $L_{1,2}$ and introduced the $\delta$-functions. Note that in some papers the last term in the left-hand side of Eq.(1) is missing. One can solve Eq.(1) and express the distribution functions $f_{\alpha \pm}$ in terms of the retarded (advanced) Green's functions which should be found from the Usadel equation. Integrating Eq.(1) once, we obtain

$$
M_{ \pm} \partial_{x} f_{ \pm}(x)+J_{S} f_{\mp}(x) \pm J_{a n} \partial_{x} f_{\mp}(x)=J_{1,2 \pm}
$$

where the indeces 1,2 relates to the intervals $\left(0, L_{1}\right),\left(L_{1}, L\right)$ and the constants $J_{1,2 \pm}$ are partial flows. For example, the partial flow $J_{1,2-}$ determines the electrical current in the intervals $\left(0, L_{1}\right),\left(L_{1}, L\right)$

$$
I_{1,2}=\sigma \int_{0}^{\infty} d \epsilon J_{1,2-}
$$

Here $\sigma$ is the conductivity of the $\mathrm{N}$ wire. As it follows from Eq.(1), the flows $J_{1,2 \pm}$ are connected with each other

$$
J_{2 \pm}-J_{1 \pm}=r A_{ \pm}
$$

The functions $r A_{ \pm}$are the flows through the $\mathrm{S} / \mathrm{N}$ interface. In particular, $r A_{-}$is the partial electrical current through the $\mathrm{S} / \mathrm{N}$ interface. The first term $\left(\nu \nu_{S}+g_{1+}\right) f_{-}$in the expression for $A_{-}$is the quasiparticle current (the term $g_{1+} f_{-}$contributes to the subgap current), and the second term $\left(g_{z+} f_{S+}+g_{z-} f_{+}\right)$is related to the supercurrent. One can show that the partial supercurrent $J_{S}$ is a constant in the interval $\left(0, L_{1}\right)$ and equals zero outside this interval.

We can solve Eqs.(2) and express the distribution functions $f_{ \pm}$through the constants $J_{1,2 \pm}$. At $x= \pm L$ the functions $f_{ \pm}$are equal to equilibrium distribution functions in the $\mathrm{N}$ reservoirs. Therefore we can find the functions $f_{ \pm}(x)$ and substituting them into Eq. $(4)$ determine $f_{ \pm}\left(L_{1}\right)$ and $J_{1,2 \pm}$. Thus the problem is reduced to solving the Usadel equation. The solution of the Usadel equation can be found either numerically or analitically in limiting cases. In the next sections we will employ this method to study the dependence of the critical current on the voltage between the $\mathrm{N}$ reservoirs and the thermoeffect. 


\section{Suppression and enhancement of the critical current}

In this section we consider a symmetrical structure shown in Fig.1a. We assume that the electric potential at the $\mathrm{N}$ reservoirs is $V$ and at the superconductors is zero (the quasiparticle currents flow from the $\mathrm{N}$ reservoirs to the superconductors and the supercurrent flows between the superconductors). The distribution functions in the reservoirs have the equilibrium form: $F_{V \pm}=[\tanh ((\epsilon+e V) \beta) \pm \tanh ((\epsilon-e V) \beta)] / 2$.

First we consider the case of a small $r$, that is, the interface resistance is large and the condition

$$
\left(\epsilon \tau_{\epsilon}\right)^{-1}<<r<<1
$$

should be satisfied. The first condition ensures that inelastic collision term can be neglected (here $\tau_{\epsilon}$ is the energy relaxation time, $\left.\epsilon \approx \min \left\{T, \epsilon_{T h}\right\}\right)$. Then the distribution function $f_{+}$is constant and in the main approximation is $f_{+} \cong\left(F_{V+}+f_{e q}\left(r_{2} \nu \nu_{s}\right)\right) /\left(1+r_{2} \nu \nu_{s}\right)$, here $r_{2}=r L_{2} / L$. The function $f_{-}$is constant in the interval $\left(0, L_{1}\right)$ and in the main approximation equals $f_{-} \cong F_{V-} /\left(1+r_{2} \nu \nu_{s}\right)$. Outside this interval the function $f_{-}$increases linearly to the value $F_{V-}$. Following the method presented above, we find the current through the $\mathrm{S} / \mathrm{N}$ interface

$$
I_{3}(V)=I_{2}-I_{1}=I_{o}(V)+I_{s g}(V) \cos \varphi-I_{c}(V) \sin \varphi
$$

The first two terms are the quasiparticle current and the last term is the Josephson supercurrent. All the components of the current depend on $V$. This expression shows that at a given control voltage $V$ and zero voltage difference between superconductors ( $\varphi$ is constant in time) the current $I_{3}$ may vary with changing $\varphi$ in some limits: $\left|I_{3}(V)-I_{o}(V)\right| \leq$ $I_{m}(V)$. This means a plateau on the $V_{S}\left(I_{3}\right)$ characteristics (see [3]); here $V_{S}=(\hbar / 2 e) \partial_{t} \varphi$ is the voltage difference between superconductors. We can write the phase-dependent part of $I_{3}$ in the form $I_{3 \varphi}=I_{m} \sin (\varphi+\alpha)$, where $I_{m}=\sqrt{I_{c}^{2}+I_{s g}^{2}}$ is the measured critical current, $\cos \alpha=I_{c} / I_{m}$. In Fig.2 we plot the dependence of the measured critical current $I_{m}$ on the control voltage $V$ for different temperatures. It is seen that the temperature dependence of the maximum of $I_{m}$ which is achieved at $e V \simeq \Delta$ is much weaker than the $I_{c}(T)$ dependence. These results qualitatively agree with the experimental data 12 .

\section{Spin injection and the critical Josephson current.}

In this section we present results of the study of spin injection on the critical Josephson current $I_{c}$ for the structure shown in Fig.1b [22]. We calculate the distribution function $f_{+}$which determines $I_{c}$ for parallel ( $\left.\uparrow\right)$ and antiparallel $(\uparrow \downarrow)$ orientations of the magnetization in the ferromagnetic $(\mathrm{F})$ reservoirs. It is assumed that the voltage $2 V$ is applied between the $\mathrm{F}$ reservoirs and a current flows between the $\mathrm{N}$ reservoirs. We consider different limits of the length of the $\mathrm{N}$ wire.

4.1. Mesoscopic limit: $L<\left\{L_{\epsilon}, L_{s p}\right\}$.

In this limit the function $f_{+} \equiv\left(f_{\uparrow_{+}}+f_{\downarrow+}\right) / 2$ does not depend on mutual orientation of ferromagnets and has the same form as in the nonmagnetic case: $f_{+}=F_{V+}$. The conductance $G$ and magnetic moment of injected spins $M$ (per unit volume of the $\mathrm{N}$ wire) depend on mutual orientation of the ferromagnetic domains. In the case of the parallel orientation ( $\uparrow$ ) we obtain $G=\left(2 R_{b \uparrow}+R_{L}\right)^{-1}+\left(2 R_{b \downarrow}+R_{L}\right)^{-1}$ and $M=0$. In the case of the antiparallel orientation ( $\downarrow)$ we have $G=2 /\left(R_{b \uparrow}+R_{b \downarrow}+R_{L}\right)$ and $M=4 \mu\left(R_{b \uparrow}-R_{b \downarrow}\right) V \beta / \sigma^{2}\left(2 R_{b \uparrow}+R_{L}\right)\left(2 R_{b \downarrow}+R_{L}\right)\left(R_{b \uparrow}+R_{2 \downarrow}+R_{L}\right)$. Here $R_{b \uparrow, \downarrow}$ and $R_{L}$ are the $\mathrm{F} / \mathrm{N}$ interface and the $\mathrm{N}$ wire resistances for electrons with up and down spins . Since the interface conductances $R_{b \uparrow, \downarrow}^{-1}$ differ from each other (they are proportional to different density of states in the ferromagnets $\nu_{F \uparrow, \downarrow}$ ), spin injection takes place in the antiparallel ( $\left.\uparrow \downarrow\right)$ configuration. However the critical current $I_{c}$ is the same for both configurations. Its dependence on $V$ is similar to that found for the structure under consideration with normal (nonmagnetic) reservoirs [6].

4.1. Semi-mesoscopic limit: $L_{\epsilon}<L<L_{s p}$.

In this limit there is no spin relaxation in the $\mathrm{N}$ wire and the magnetization $M$ is determined again by formulae presented above. On the other hand the magnetization is related to a potential $V_{s p}$ which determines an imbalance between the spin subsystems in the $\mathrm{N}$ wire: $M=2 \mu \nu e V_{s p}$. The function $f_{+} \equiv\left(f_{\uparrow+}+f_{\downarrow+}\right) / 2$ has an equilibrium form corresponding to the potential $V_{s p}: f_{+}=\left[\tanh \left(\epsilon+e V_{s p}\right) \beta+\tanh \left(\epsilon-e V_{s p}\right) \beta\right] / 2$. Therefore in the case of parallel $(\uparrow \uparrow)$ orientation $I_{c}$ does not depend on $V$ as $V_{s p}=0\left(V\right.$ is proportional to $\left.V_{s p}\right)$. In the case of antiparallel ( $\downarrow$ ) orientation $I_{c}$ does depend on $V_{s p}$ in the same way as in the section 4.1. A similar effect of spin injection into a supereconductor on the energy gap $\Delta$ was analysed in Ref. [23]. The critical Josephson current is more sensitive to the form of the distribution function than the energy gap $\Delta$. Therefore the effect considered here allows one to realise the $\pi-$ junction and to make certain conclusions about the energy and spin relaxation rate.

\section{Giant thermoelectric effect}

It is well known that if the terminals of a normal conductor are maintained at different temperatures $\delta T=T_{o} \pm \delta T$, then in the absence of the current an thermoemf $V_{e m f}$ appears at the terminals. The magnitude of $V_{e m f}$ is equal 
to: $e V_{e m f}=c_{1}\left(T / \epsilon_{F}\right) \delta T$, where $c_{1}$ is a factor of the order 1 and $\epsilon_{F}$ is the Fermi energy. In this report we study the thermoelectric effect in the mesoscopic structure shown in Fig.1a. It will be shown that a temperature difference between the normal $(\mathrm{N})$ reservoirs leads to a voltage between normal and superconducting curcuits $V_{T}$. The magnitude of this voltage does not contain the small parameter $\left(T / \epsilon_{F}\right)$, and besides it oscillates with a variation of the phase difference $\varphi$ between the superconductors.

We assume that the superconductors are connected via a superconducting loop and the phase difference between them $\varphi$ is controlled by an applied magneitic field. The $\mathrm{N}$ reservoirs are disconnected and maintained at different temperatures $T( \pm L)=T_{o} \pm \delta T$. We will calculate the electric potential in the $\mathrm{N}$ film and in particular the potential $V_{T}$ in the N reservoirs. Since we set the potential in the superconductors equal to zero, the potential $V_{T}$ is the voltage difference between the $\mathrm{N}$ reservoirs and superconductors which arises in the presence of the temperature difference $\delta T$. In the limit of high interface resistance (see condition (5)) we obtain from Eq.(2) in the main approximation in $r: f_{+}(x) \cong J_{+} x+f_{+}(0)$, where $f_{+}(0)=f_{e q} \equiv \tanh \left(\beta_{o} \epsilon\right)$, and $J_{+}=\delta \beta \epsilon / L \cosh ^{2}\left(\beta_{o} \epsilon\right), \delta \beta=-\beta \delta T / T_{o}$. We assumed the ratio $\delta T / T_{o}$ to be small and expant the distribution functions in the $\mathrm{N}$ reservoirs.

In order to find the function $f_{-}$, we consider Eq.(2) and take into account that $J_{2-}$ is zero (no current through the $\mathrm{N}$ reservoirs). In the absence of the temperature gradient we obtain from this equation

$$
f_{-}=0, \quad J_{S} f_{e q}=J_{1 e q}=r\left(g_{z-}+g_{z+}\right) f_{e q}
$$

The temperature gradient leads to the non-zero function $f_{-}(x)$ which determines the electrical potential $V_{T}(x)$. We find from Eq.(2)

$$
f_{-}(x)=f_{-}(0)+\delta J_{1} x+\int_{0}^{x} d x_{1}\left[J_{a n} \partial_{x} \delta f_{+}\left(x_{1}\right)-J_{s} \delta f_{+}\left(x_{1}\right)\right]
$$

where $\delta f_{+}(x) \equiv f_{+}(x)-f_{e q}$. From Eq.(1) we obtain for $\delta J_{1} \equiv J_{1}-J_{1 e q}$

$$
\delta J_{1}= \pm r\left[g_{z+} \delta f_{+}\left( \pm L_{1}\right)-g_{1+} f_{-}\left( \pm L_{1}\right)\right]
$$

Here the signs \pm relate to the points $\pm L_{1}$. From Eqs.(8) and (9) it follows that $\delta J_{1}=0$, and in the main approximation in $r$ the distribution function is $f_{-}(0) \approx \delta f_{+}\left( \pm L_{1}\right) g_{z+} / g_{1+}$, where $\delta f_{+}\left( \pm L_{1}\right)= \pm J_{+} L_{1}$. The electric potential $V_{T}$ is related to the function $f_{-}$and is an even function of $x$

$$
e V_{T}=\int_{0}^{\infty} d \epsilon f_{-}=\frac{L_{1}}{L} \frac{\delta T}{T_{o}} \beta \int_{0}^{\infty} d \epsilon \frac{g_{z+}}{g_{1+}} \frac{\epsilon}{\cosh ^{2}\left(\beta_{o} \epsilon\right)}
$$

Here we substitute the function $f_{-}(0)$ which is the main contribution to the function $f_{-}(x)$. Therefore the potential $V_{T}$ arising in the presence of the temperature gradient is almost constant along the $\mathrm{N}$ wire. This potential equals approximately the voltage difference $V_{T}$ between the $\mathrm{N}$ reservoirs and superconducting loop. It is worth noting that $V_{T}$ determined by Eq.(10) does not depend on the small parameter $r$ (however one should have in mind that the condition (5) imposes limits on this parameter). We calculated the integrand using both analytical an numerical solutions of the Usadel equation. In Fig.3 we show the temperature dependence of $V_{T}$ for $\varphi=\pi / 2$. We see that the dependence $V_{T}(T)$ is nonmonotonic (reentrant behaviour). One can easily estimate $V_{T}$ on the order of magnitude. We obtain

$$
e V_{T}=\delta T\left(L_{1} / L\right) \sin \varphi \begin{cases}\left(T / \epsilon_{T h}\right) C_{1}(\varphi), & T<<\epsilon_{T h} \\ \left(\epsilon_{T h} / T\right)^{2} C_{2}(\varphi), & T>>\epsilon_{T h}\end{cases}
$$

Here $C_{1,2}(\varphi)$ are periodic functions of the phase difference $\varphi$; they are of order 1 and are not zero at $\varphi=0$. If the ratio $T / \epsilon_{T h}$ is of order 1 , then the thermoemf is of the order $\delta T$, that is, several orders greater than the thermoemf in the normal metals.

The physical explanation of the effect is the following. The temperature gradient creates a deviation of the distrubtion function $\delta f_{+}=-\delta(n+p)$ from equilibrium. On the other hand the superconductors do not affect this function because complete Andreev reflections conserve the total number of excess electrons and holes. The function $\delta f_{+}$has different signs at $\pm L_{1}$ and leads to an additional Josephson current $\delta J_{S}=r g_{z+} \delta f_{+}\left(L_{1}\right)$ of the same sign at $\pm L_{1}$ (sign of the function $g_{z+}$ is different at these points). In order to compensate these additional currents, the potential $V_{T}$ arises in the $\mathrm{N}$ wire producing a subgap current $\delta J_{s g}=r g_{1+} f_{-}\left( \pm L_{1}\right)$ which compensates the current $\delta J_{S}$.

We have neglected the ordinary thermoelectric current $I_{T}$ in the $\mathrm{N}$ wire because it contains a small parameter $\left(T / \epsilon_{T}\right)$ and leads to a small contribution to $V_{T}$. Recently the influence of the proximity effect on the ordinary thermoelectric 
effect in the mesoscopic S/N structure was studied both theoretically [24] and experimentally [25]. The effect analysed here is much stronger.

In simplified models we have analysed three possible effects in the 4-terminals $\mathrm{S} / \mathrm{N}$ mesoscopic structures; one of them (see section 3) has been observed experimentally. Further theoretical studies of these and other effects are needed to account for processes which were neglected in our analysis. It is interesting, for example, to investigate how the obtained results are changed by the energy relaxation in the $\mathrm{N}$ wire which seems to be faster than it was expected 26

We are grateful to the EPSRC for their financial support.

[1] B. J. Van Wees, K.-M. H. Lenssen, C.J.P.M. Harmans, Phys.Rev.B 44, 470 (1991).

[2] A.F. Volkov, Phys. Rev. Lett. 74,4730(1995); JETP Lett, 61,565 (1995)

[3] A.F.Volkov and H.Takayanagi, Phys. Rev. 56, 11184 (1997); A.F.Volkov and V.V.Pavlovskii, JETP Lett. 64, 670 (1996)

[4] R.Seviour and A.F.Volkov, Phys.Rev 61 (13) (2000) (in press)

[5] A.F.Volkov, R.Seviour, and V.V.Pavlovskii, Superlattices and Microstructures 25, 647 (1999)

[6] S.K. Yip, Phys.Rev. 58, 5803 (1998)

[7] F.K. Wilhelm, G. Schön and A.D. Zaikin, Phys. Rev. Lett. 81, 1682(1998)

[8] W.Belzig, F.K.Wilhelm, C.Bruder, G.Schoen, and A.D.Zaikin, Superlattices and Microstructures 25, 1251 (1999).

[9] G.Wendin and V.S.Shumeiko, J. of Phys.and Chemistry of Solids 56, 1773 (1995); Phys.Rev.B53, R6006 (1996); Superlattices and Microstrutures 25, 983 (1999).

[10] Li-Fu Chang and P.F. Bagwell, Phys. Rev. B 55, 12678 (1997)

[11] J.J. Baselmans, A.F. Morpurgo, T.M. Klapwijk and B.J. van Wees Nature 397, 43 (1999)

[12] J.Kutchinsky et al. Phys.Rev.Lett. 83, 4856 (1999).

[13] A.I. Larkin and Yu.N. Ovchinnikov, in Nonequilibrium Superconductivity, ed. by D.N. Langenberg and A.I. Larkin (Elsevier, Amsterdam, 1986), p.493.

[14] S.N.Artemenko, A.F.Volkov and A.V.Zaitsev, Zh. Eksp. Teor. Fiz. 76, 1816 (1979)

[15] S.N.Artemenko, A.F.Volkov and A.V.Zaitsev,Solid State Comm. 30, 771 (1979)

[16] A.V.Zaitsev, Sov. Phys JETP Lett. 51, 41 (1990); Physica C185-189, 2539(1991)

[17] A.F.Volkov, A.V.Zaitsev, and T.M.Klapwijk, Physica C210, 21 (1993)

[18] Yu.V. Nazarov, Phys. Rev. Lett. 73,1420 (1994)

[19] L.G.Aslamazov and A.I.Larkin, Zh. Eksp. Teor. Fiz. 70, 1314 (1976)

[20] L.G.Aslamazov and A.F.Volkov, in Nonequilibrium Superconductivity, ed. by D.N. Langenberg and A.I. Larkin (Elsevier, Amsterdam, 1986), p.65.

[21] A.Schmid and G.Schoen, J.Low Temp.Phys. 20, 207 (1975)

[22] A.F.Volkov and V.I.Falko (unpublished)

[23] S.Takahashi, H.Imamura, and S.Maekava, Phys.Rev.Lett. 82, 3911 (1999)

[24] N.R.Claughton and C.J.Lambert, Phys. Rev. B53, 6605 (1996).

[25] J. Eom, C.J. Chien, V. Chandrasekhar,Superlattices and Microstructures 25, 733 (1999)

[26] H.Pothier, S.Gueron, N.O.Birge, D.Esteve, and M.H.Devoret, Phys.Rev.Lett.79, 3490 (1997) 

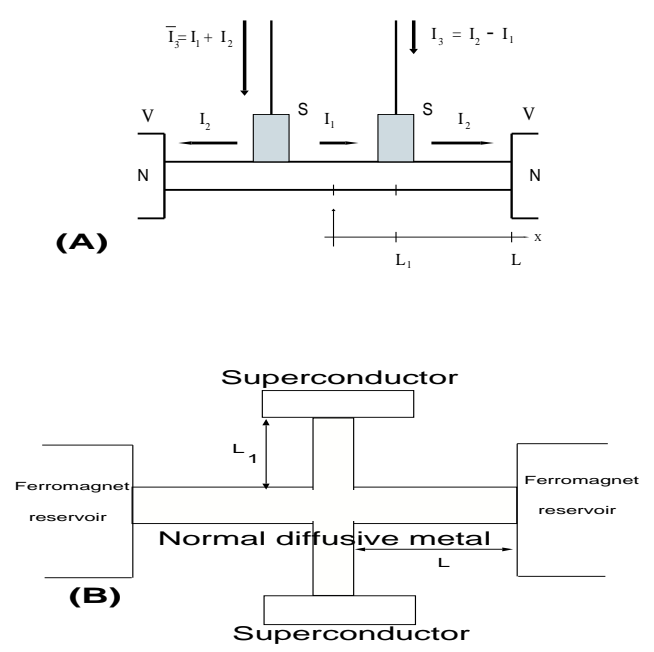

FIG. 11 Schematic view of the 4-terminal S/N/S structure under consideration.

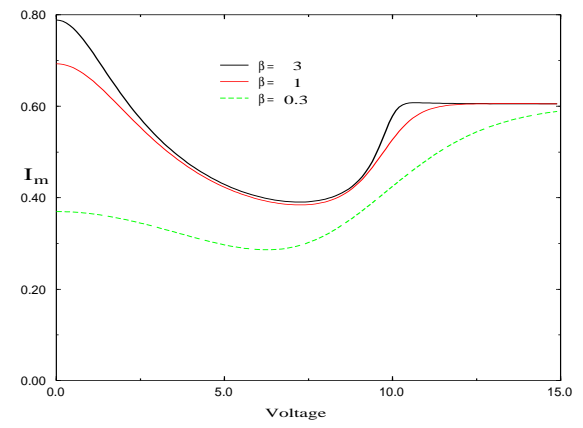

FIG. 2 The measured critical current $\left(I_{m}\right)$ vs $V$ for different temperatures: $\beta=\epsilon_{T h} / 2 T$. The parameters are: $\Delta=$ $10 \epsilon_{T h}, L_{1} / L=0.3, r=0.3$.

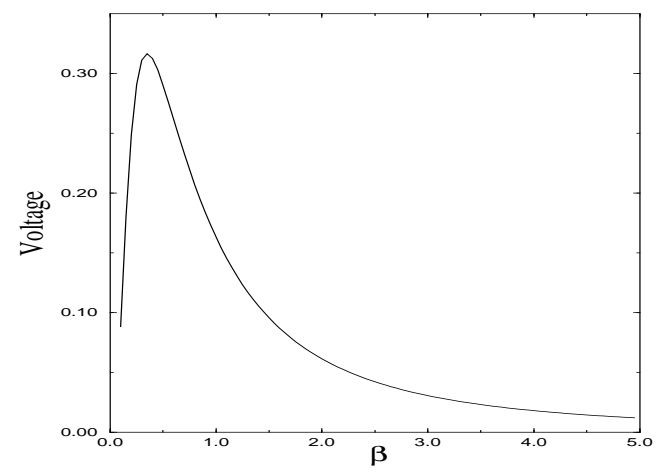

FIG. 3 The dependence of the normalised voltage $\widetilde{V_{T}}$ on inverse temperature $\beta$ at $\varphi=\pi / 2\left(\right.$ the parameters are $\Delta / \epsilon_{T h}=$ $\left.10, L_{1} / L=0.5\right)$. 